A. Pramesh Rao, G. Swarup and Gopal-Krishna, eds.

\title{
Low Frequency Emission Regions in Pulsars
}

\author{
Jarosław Kijak \\ J. Kepler Astronomical Center, Pedagogical University, Lubuska 2, \\ PL-65-265 Zielona Góra, Poland
}

\begin{abstract}
The systematic increase of component separation and profile widths with decreasing frequency in pulsars suggests that the radiation at different frequencies is emitted from different altitudes above the polar cap. This concept is known as the radius-to-frequency mapping. According to RFM the observed low-frequency radiation should be generated farther from the neutron star than higher-frequency radiation. We discuss radial locations of the pulsar emission regions using a geometrical method for the estimation of emission altitudes. It is argued that the emission altitude at a given frequency is slightly different for young and old pulsars.
\end{abstract}

\section{Emission Altitude}

We have studied the concept of RFM using the pulse width method of estimation to multifrequency data (Kijak \& Gil 1997; Kijak \& Gil 1998). The semi-empirical relationship for the pulsar radio emission altitudes

$$
r_{\mathrm{KG}}=(55 \pm 5) R \nu_{\mathrm{GHz}}^{-0.21 \pm 0.07} \tau_{6}^{-0.07 \pm 0.03} P^{0.33 \pm 0.05}
$$

suggests that the very low frequency emission originates, in typical pulsars, at altitudes lower than a few thousand kilometres above polar cap. In the millisecond pulsars the emission region is much closer to the polar cap (see Fig. 4 in Kijak \& Gil 1998). In general, emission region is very compact and lies near the surface of the neutron star, apparently closer than 10 per cent of the light-cylinder radius. It is significant that this result is consistent with results obtained using different estimation methods (Gupta et al. 1999 and see also Table 4 in Kijak \& Gil 1998).

\section{Young and Old pulsars}

In previous work, the dependence of emission altitude on the timing age was not obvious, although some evidence existed. Now, we present analysis of emission altitudes at $1.4 \mathrm{GHz}$ (Fig. 1) for a number of young (below 1 million years) and very old (above 1 billion years) pulsars for which both pulse width measurement (for a high-quality profile) and estimation of the inclination angle (between the rotation and magnetic axes) and the impact angle (of the closest approach of the observer to the magnetic axis) were available. This analysis shows that the age dependence may really exist. From our data we obtained $r_{\mathrm{em}}\left(\tau_{6}\right) \propto \tau_{6}^{-0.05 \pm 0.03}$. 


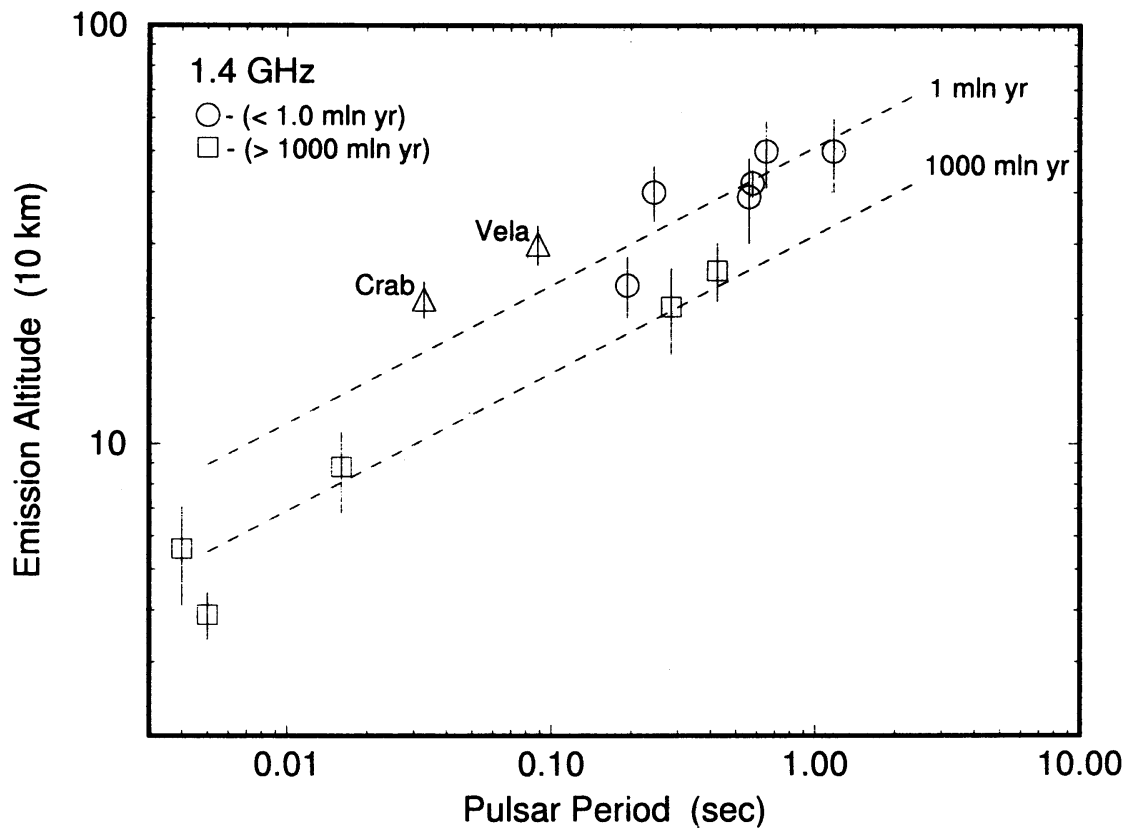

Figure 1. Analysis of emission altitudes for young and old pulsars at 1.4 $\mathrm{GHz}$. The dashed lines correspond to different values of timing age $\tau_{6}$ from equation (1).

\begin{tabular}{llll} 
B0531+21MP & B $0833-45$ & & very young PSRs \\
\hline B0540+23 & B1749-28 & B1915+13 & $\sim 1$ milion \\
B1916+14 & B1924+16 & B1933+16 & years \\
\hline J1022+1001 & $\mathrm{J} 1713+0747$ & $\mathrm{~B} 1848+04$ & very old \\
B1855+09MP & B1952+29 & & PSRs
\end{tabular}

Thus we confirm that indeed the emission altitude depends on pulsar period $P$, frequency $\nu$ and pulsar age $\tau_{6}$ (in units of $10^{6} \mathrm{yr}$ ) in a way which can be decribed by our equation (1).

Acknowledgments. The work described here was supported by the KBN Grant 2 P03D 01512.

\section{References}

Gupta, Y., Bhat, N. D. R., Rao, A. P. 1999, ApJ, 520, 173

Kijak, J., Gil, J. 1997, MNRAS, 288, 631

Kijak, J., Gil, J. 1998, MNRAS, 299, 855 